\title{
Abnormal Expression and Distribution of MMP2 at Initial Stages of Alzheimer's Disease-Related Pathology
}

\author{
Beatrice Terni ${ }^{\mathrm{a}, \mathrm{b}, *}$ and Isidro Ferrer ${ }^{\mathrm{a}, \mathrm{c}, \mathrm{d}}$ \\ ${ }^{a}$ Institute of Neuropathology, Bellvitge University Hospital, Hospitalet de Llobregat, Spain \\ ${ }^{\mathrm{b}}$ Laboratory of Neurobiology, Bellvitge Biomedical Research Institute (IDIBELL), Hospitalet de Llobregat, Spain \\ ${ }^{\mathrm{c}}$ University of Barcelona, Hospitalet de Llobregat, Spain \\ ${ }^{\mathrm{d}}$ CIBERNED, Institute Carlos III, Ministry of Health, Spain
}

Handling Associate Editor: Irina Alafuzoff

Accepted 3 March 2015

\begin{abstract}
Previous studies have shown that metalloproteinases (MMPs) participate in the clearance of amyloid- $\beta$ (A $\beta$ ) in Alzheimer's disease (AD); MMP2 and MMP3 cleave soluble A $\beta$, and both MMP9 and MT1-MMP are able to degrade soluble and fibrillar forms of $\mathrm{A} \beta$. The present study shows increased expression levels of active MMP2 in the entorhinal cortex at early stages of AD-related pathology (Braak and Braak stages I/II-0 and III/IV-A) as revealed by western blotting and gelatin zymography. Confocal microscopy discloses co-localization of MMP2 and phospho-tau in neurofibrillary tangles and dystrophic neurites. MMP2 has the capacity to cleave recombinant tau in vitro in a dose-dependent manner, consistent with a physiological function of MMP2 in normal tau proteolysis. However, MMP2 does not cleave hyperphosphorylated and dephosphorylated tau from enriched paired helical filament fractions. These observations raise the possibility that accumulation of MMP2 in neurofibrillary tangles and concomitant loss of proteolytic capacity on tau protein is a response geared to eliminating production of toxic truncated tau species in $\mathrm{AD}$ brains.
\end{abstract}

Keywords: Alzheimer's disease, metalloproteinases, MMP2, neurofibrillary tangles, tau

\section{INTRODUCTION}

Alzheimer's disease (AD) is a progressive neurodegenerative disease causing loss of neurons and neuronal connectivity in several brain regions. In addition, multiple metabolic pathways are altered in the course of the degenerative process $[1,2]$. AD is a long-lasting biological process in which neurofibrillary tangles (NFTs) progress from the entorhinal and transentorhinal cortex (stages I and II), hippocampus and limbic system (stages III and IV), and the

\footnotetext{
${ }^{*}$ Correspondence to: Beatrice Terni, Laboratory of Neurobiology, Bellvitge Biomedical Research Institute (IDIBELL), Pavelló del Govern, Feixa Llarga s/n, 08907 Hospitalet de Llobregat, Spain. Tel: +34 934024279; Fax: +34 934035810; E-mail: bterni@idibell.cat.
}

whole neocortex (V and VI), whereas the progress of amyloid- $\beta$ (A $\beta$ ) plaques follows a different regional pattern with increasing $A \beta$ plaque burden in the whole brain [3].

The metabolism of $A \beta$ production and clearance, and hyperphosphorylation of tau, are very complex and involve several mechanisms, some leading to the formation of oligomeric and fibrillar species and truncated products which are toxic to nerve cells $[4,5]$.

A growing body of evidence indicates that matrix metalloproteinases (MMPs) may play an important role in the pathogenesis of $\mathrm{AD}$ and other neurodegenerative pathologies. MMPs, collectively called matrixins, are a family of zinc and calcium enzymes that are able to digest components of the extracellular matrix such as collagen, proteoglycan, and laminin [6]. 
The distribution of the MMPs in the central nervous system is widespread; they are secreted by neurons, oligodendrocytes, microglia, and endothelial cells [7]. MMPs participate in the clearance of $\mathrm{A} \beta$ in $\mathrm{AD}$ [8-11]. In particular, growing data point to MMP2 (gelatinase A) and MMP9 (gelatinase B) as important players in the metabolism of $A \beta$. Upregulation of MMP2 and MMP9 is induced by $A \beta$ in mixed primary culture $[12,13]$. A $\beta P P / P S 1$ transgenic mice have increased levels of MMP2 and MMP9 in astrocytes around $A \beta$ plaques; and treatment with the MMP inhibitor GM6001 increases $A \beta$ levels in brain interstitial fluid in these transgenic mice [14]. Although most evidence on the role of MMPs in AD points to advanced cases of the disease, some data indicate that deregulation of metalloproteinases may also occur at early stages of AD [15]. Recent studies clearly demonstrated the upregulation of MMP2 and MMP9 in the hippocampus of the $5 \mathrm{xFAD}$ mouse model of AD during the asymptomatic and initial stage of the pathology [16].

We hypothesized that MMP2 and MMP9 may participate in the normal metabolism of other proteins involved in $\mathrm{AD}$, in addition to $\mathrm{A} \beta$, and particularly in tau metabolism. Recently, it has been shown that limited cleavage by MMP9 enhances the formation of tau oligomers [17]. Furthermore, we speculated that the effects of MMP deregulation at early stages of AD-related pathology may have implications in tau proteolysis. Therefore, in this study, we started investigating modifications in the expression and localization of MMP2 in the entorhinal cortex in normal middleaged individuals with no AD-related pathology and in cases with early stages of AD-related pathology mainly consistent with the presence of NFTs. Functional studies assessing the capacity of MMP2 to cleave recombinant tau, along with hyperphosphorylated and dephosphorylated tau aggregates extracted from cortex of AD, were carried out to increase understanding of the contribution of MMP2 in tau metabolism.

\section{MATERIALS AND METHODS}

\section{Tissue samples}

The selection of cases examined in the present study corresponded to a consecutive series of brain donations of patients dying from unrelated causes at a general hospital. The postmortem delay between death and processing was between 4 and $6.30 \mathrm{~h}$. Cases with neurological symptoms and signs, hepatic or renal function impairment, hypoxia, infection, autoimmune disorders, disseminated neoplasia, or prolonged agonic state were excluded. The clinical records in all cases with AD-related pathology were re-assessed by phone calls or interviews with relatives asking about any neurological or cognitive change. Therefore, all cases with AD-related pathology were considered possible preclinical stages. At autopsy, half of each brain was fixed in formalin, while the other half was cut in coronal sections $1 \mathrm{~cm}$ thick, and selected brain regions were dissected, collected, and numbered in plastic bags, frozen on dry ice, and stored at $-80^{\circ} \mathrm{C}$ until use. For diagnostic morphological studies, the brains were fixed by immersion in $4 \%$ buffered formalin for 2 or 3 weeks. The tissue was embedded in paraffin, and de-waxed sections, $5 \mu \mathrm{m}$ thick, were processed for neuropathological study. Staging of AD-related pathology was carried out following Braak and Braak criteria [3], adapted to paraffin sections [18]. Cases with additional pathology (either tau, i.e., grains; $\alpha$-synuclein: Lewy bodies in other brain regions; TDP-43 pathology; or vascular in any area) were excluded. Special care was also taken to minimize the amount of $A \beta$ plaques in the present series to increase the weight of tau pathology. Following these conditions, the following cases were used in the present study: middle-aged subjects with no neuropathological lesions (MA): 9 (8 men, 1 women; age: 53.6 \pm 9.3$)$, AD-related pathology stages I/II-0:7 (3 men, 4 women; age: $66 \pm 7.5)$, and ADrelated pathology stages III/IV-A: 8 (4 men, 4 women, age: $79 \pm 5.3)$. Studies were focused on the entorhinal cortex.

\section{Preparation of samples}

$80 \mathrm{mg}$ of entorhinal cortex from MA, ADI/II-0, and ADIII/IV-A cases was homogenized in $800 \mu \mathrm{l}$ of Ripa buffer (50 mM Tris $\mathrm{HCl} \mathrm{pH} 8 ; 150 \mathrm{mM} \mathrm{NaCl}$, $1 \%$ NP-40; $0.5 \%$ sodium deoxycholate; $0.1 \%$ SDS) plus $1 \mathrm{mM}$ phenylmethylsulfonyl fluoride (PMSF) and protease inhibitor tablets (Roche Molecular Systems, Barcelona, Spain). The samples were then centrifuged at $10,000 \times \mathrm{g}$ for $10 \mathrm{~min}$ and the total protein of the supernatant was quantified with the BCA (bicinchoninic acid) assay (Biorad, CA, USA).

\section{Western blot}

$20 \mu \mathrm{g}$ of total protein was run in SDS-PAGE using $8 \%$ acrylamide gel and then transferred to a nitrocellulose membrane for $2 \mathrm{~h}$ at $200 \mathrm{~mA}$. The membrane was then blocked with $5 \%$ skimmed milk for $1 \mathrm{~h}$ prior to overnight incubation at $4{ }^{\circ} \mathrm{C}$ with anti-MMP2 monoclonal antibody (Millipore-Merck, Barcelona, Spain) at a dilution of 1:1,000. The blots 
were then incubated for $2 \mathrm{~h}$ at room temperature with anti-mouse horseradish conjugated secondary antibody (DAKO, Barcelona, Spain) and visualized with the ECL chemiluminescence method (Amersham, Barcelona, Spain). The monoclonal anti- $\beta$-actin antibody (Sigma-Aldrich, Madrid, Spain) was used as a control of protein loading. Protein expression levels were determined by densitometry of the bands using Image $\mathbf{J}$ software. Measurements were expressed as arbitrary units and the results were normalized for $\beta$-actin. The numerical data were statistically analyzed using ANOVA test.

\section{In gel zymography}

Gelatinolytic activity of MMP2 was measured with in gel zymography assay following a protocol adapted from one previously described [14]. Briefly, $60 \mathrm{mg}$ of entorhinal cortex from MA, ADI/II-0, and ADIII/IV-A cases was homogenized in Lysis buffer (50 mM Tris- $\mathrm{HCl} \mathrm{pH} 7.6,150 \mathrm{mM} \mathrm{NaCl}, 5 \mathrm{mM} \mathrm{CaCl}_{2}$, $0.5 \%$ Triton, $0.05 \%$ Brij-35) along with $1 \mathrm{mM}$ PMSF and protease inhibitors, and left for $30 \mathrm{~min}$ in agitation at $4{ }^{\circ} \mathrm{C}$. The supernatant was collected by centrifugation at $10,000 \times \mathrm{g}$ for $10 \mathrm{~min}$ and the total amount of protein was quantified with BCA method. The same amount of protein from each case was incubated with $50 \mu \mathrm{l}$ of gelatin-Sepharose 4B (Pharmacia Biotech, Uppsala, Sweden) for $1 \mathrm{~h}$ with constant shaking at $4^{\circ} \mathrm{C}$. After centrifugation, the gelatin sepharose pellet was washed twice with lysis buffer and then incubated with Elution buffer (lysis buffer plus 10\% DMSO) for $30 \mathrm{~min}$ in agitation at $4{ }^{\circ} \mathrm{C}$. $30 \mu \mathrm{l}$ of the eluted material containing gelatinases was mixed with an equal volume of $2 \mathrm{X}$ sample buffer $\mathrm{pH} 6.8$ and run at $30 \mathrm{~mA}$ in a $10 \%$ SDS-PAGE gel containing $0.1 \%$ gelatin. After running, the gel was washed twice with $2.5 \%$ Triton for $20 \mathrm{~min}$, equilibrated for $15 \mathrm{~min}$ in the activity buffer (50 mM Tris- $\mathrm{HCl} \mathrm{pH} 7.5,10 \mathrm{mM} \mathrm{CaCl}_{2}, 0.025 \mathrm{NaN}_{3}$ ) and finally incubated in the same buffer for $20 \mathrm{~h}$. The gel was then stained for $1 \mathrm{~h}$ with $0.5 \%$ Coomassie brilliant blue in $40 \%$ methanol and $10 \%$ acetic acid, and destained until bands clearly appeared. The bands corresponding to the proteolytic activity of MMP2 were quantified using the Image J software and the data corresponding to $\mathrm{MA}$ and $\mathrm{AD}$ and cases were statistically analyzed using ANOVA test.

\section{Double-labeling immunofluorescence and confocal microscopy}

$5 \mu$ l-thick sections from entorhinal cortex were first de-waxed and then stained with a saturated solution of
Sudan black for 10 min to block the autofluorescence of lipofuscin. After one wash in $70 \%$ ethanol and several in distilled water, the sections were blocked for $1 \mathrm{~h}$ with $10 \%$ fetal bovine serum (FBS) in PBS and incubated overnight at $4{ }^{\circ} \mathrm{C}$ with $1 \%$ FBS/PBS solution containing a combination of mouse anti-MMP2 (Abcam, Clone 4D3, Cambridge, UK) and rabbit antiTau-Thr181 (Calbiochem, Barcelona, Spain) primary antibodies used at 1:50 and 1:100 dilutions, respectively. After washing with PBS, the sections were incubated for $45 \mathrm{~min}$ in the dark at room temperature with a cocktail of Alexa488 anti-mouse and Alexa555 anti-rabbit secondary antibodies at a dilution of 1:400 in the same vehicle solution of primary antibodies. Nuclei were stained with DRAQ5 (Molecular Probes, Oregon, USA) diluted 1:2,000 for $10 \mathrm{~min}$. Some sections were incubated only with the secondary antibodies. In addition, some samples were pre-incubated with recombinant MMP2 previously boiled to expose the antibody epitopes. The antigenantibody mixture was made at 10:1 ratio in PBS and left overnight at $4{ }^{\circ} \mathrm{C}$ with gentle shaking. Sections from the same individual were then incubated with either MMP2 antibody alone or pre-absorbed MMP2 antibody in a parallel experiment. After washing in PBS, the sections were finally mounted in Immuno-Fluore Mounting medium (ICN Biomedicals), sealed, and dried overnight. Sections were examined with a Leica TCS-SL confocal microscope. Sections pre-incubated with the antigenic peptide and sections incubated only with the secondary antibodies were negative.

\section{Cleavage of recombinant tau by $M M P 2$}

Full-length MMP2 (Sigma) was diluted to a final concentration of $100 \mu \mathrm{g} / \mathrm{ml}$ and activated through its incubation with $1 \mathrm{mM}$ APMA (4-aminophenylmercuric acetate) (Sigma) for $1 \mathrm{~h}$ at $37^{\circ} \mathrm{C}$. Afterwards, $2 \mu \mathrm{g}$ of the longest form of recombinant tau, which includes exons 2 and 3, and four microtubules-binding repeats (T40) (r-peptide) were incubated with $50 \mathrm{ng}, 200 \mathrm{ng}$, and $500 \mathrm{ng}$ of activated MMP2 in a buffer containing $50 \mathrm{mM}$ Tris- $\mathrm{HCl} \mathrm{pH} \mathrm{7.5,}$ $10 \mathrm{mM} \mathrm{CaCl}_{2}$, and $150 \mathrm{mM} \mathrm{NaCl}$. The reaction was left at $37^{\circ} \mathrm{C}$ for $3 \mathrm{~h}$ and then stopped by the addition of Laemmli SDS sample buffer and followed by boiling. The samples were subjected to $12 \%$ SDS-PAGE and the tau proteolytic profile was visualized first on silver staining (Amersham) and then by immunoblotting with both N-terminal tau (Millipore) and Tau 46 C-terminal (Abcam) antibodies diluted 1:1,000. 
A
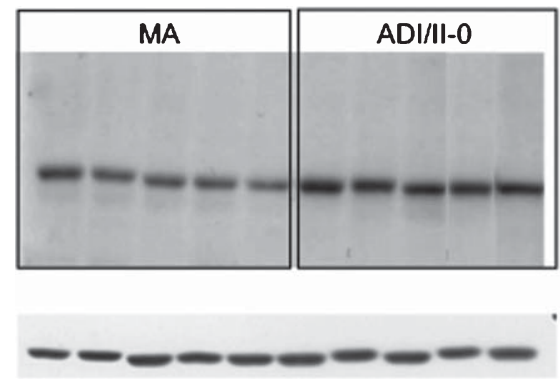

B

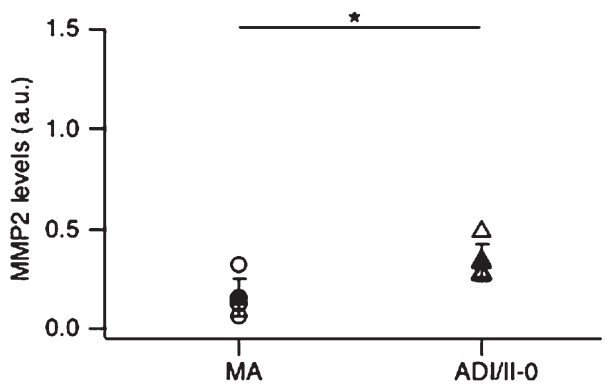

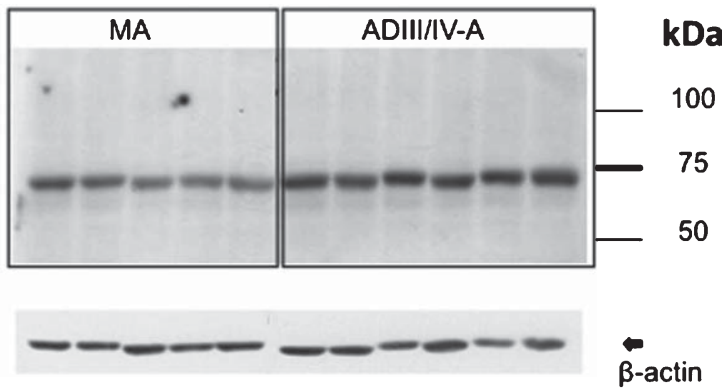

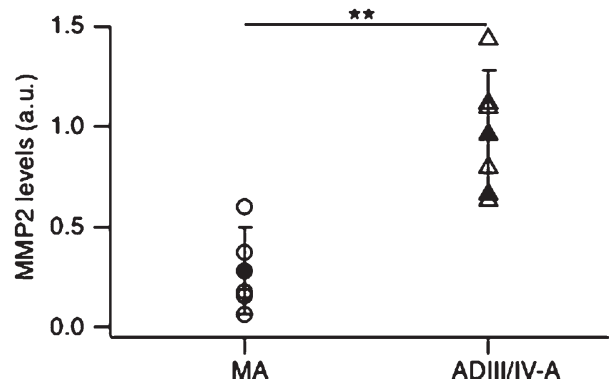

Fig. 1. Immunoblots of entorhinal cortex homogenates of $(n=5)$ AD cases stages I/II-0 (left panel); $(n=6)$ AD cases stages III/IV-A (right panel) and $(n=5)$ middle-aged (MA) cases with no AD-related pathology are incubated with antibodies to MMP2. Western blot densitometry (B) shows increment of MMP2 expression in ADI/II-0 $\left({ }^{*} p=0.021\right)$ and ADIII/IV-A $\left({ }^{* *} p=0.0027\right)$ compared to MA subjects. Data values of MMP2 were normalized to $\beta$-actin. Open circle and triangles show values of individual cases. Filled shapes indicate mean \pm SD.

\section{PHF cleavage by MMP2}

Paired helical filaments (PHFs) made of hyperphosphorylated tau were extracted as previously described [19]. The samples were incubated with or without E. coli alkaline phosphatase (18 Units/ml, Sigma) at $68^{\circ} \mathrm{C}$ for $3 \mathrm{~h}$. After this treatment, half of the sample was incubated with $500 \mathrm{ng}$ of activated MMP 2 at $37^{\circ} \mathrm{C}$ for $3 \mathrm{~h}$. The reaction was stopped by adding Laemmli SDS sample buffer followed by boiling. The other half was immediately mixed with Laemmli SDS sample buffer and boiled. The samples were subjected to $12 \%$ SDS-PAGE and immunoblotted with N-terminal tau and Tau46 C-terminal antibodies.

\section{RESULTS}

Levels of MMP2 are increased in entorhinal cortex at early stages of AD-related pathology

Total homogenates from entorhinal cortex from MA, ADI/II-0, and ADIII/IV-A cases were run in SDS-PAGE gels and immunoblotted with antibodies recognizing full-length MMP2 (gelatinase A). The monoclonal antibody for MMP2 revealed an increment of this protein in ADI/II-0 and ADIII/IV-A with respect to MA cases (Fig. 1A, B; Supplementary Fig. 1). Values of MMP2 were normalized to $\beta$-actin; ANOVA test, used to compare the $\mathrm{AD}$ groups with MA group, produced $p$-values for ADI/II-0 and ADIII/IV-A of 0.021 and 0.0027 , respectively.

To further investigate the possible alteration of this metalloprotein at early stages of AD-related pathology, MMP2 was isolated from entorhinal cortex. The gelatin zymography assay showed one typical band of $72 \mathrm{kDa}$ corresponding to MMP2 (Fig. 2). A significant increase in MMP2 gelatinolytic activity was observed in ADI/II-0 $(p=0.007)$ and ADIII/IV-A $(p=0.008)$ groups with respect to MA cases with no AD-related pathology (Fig. 2A, B), in agreement with the difference in MMP2 expression levels shown by western blot.

Immuno-co-localization of MMP2 with tau lesions in the entorhinal cortex at early stages of AD-related pathology

MMP2 is weakly expressed in neurons in the normal brain; however, MMP2 appears to accumulate in neurons containing NFTs in AD. Sections of entorhinal cortex of AD and MA cases were double stained with anti-MMP2 and anti-phosphoTau-Thr181 antibodies 
A
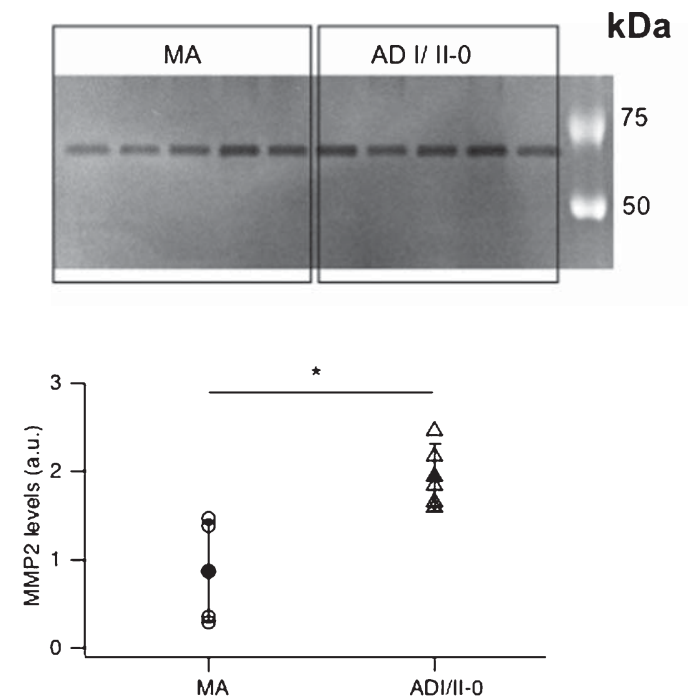

B
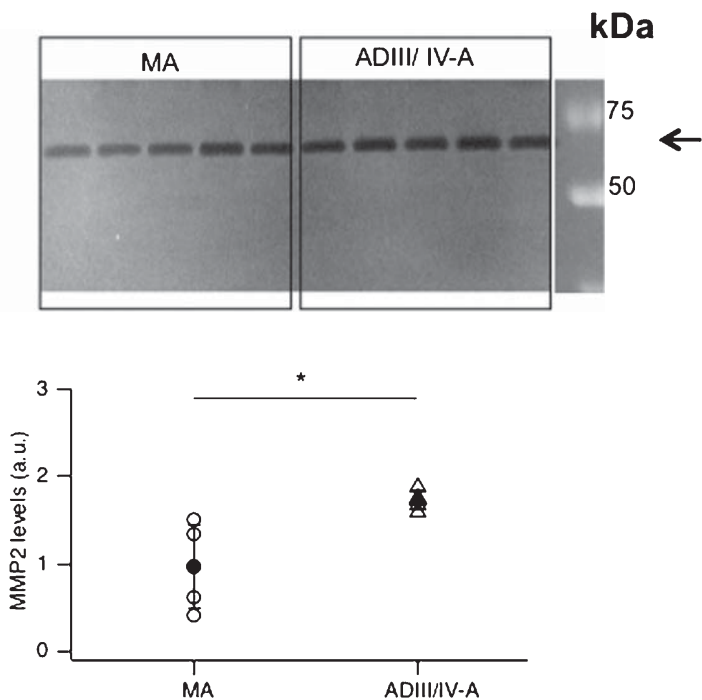

Fig. 2. Gelatinases are isolated from entorhinal cortex of ADI/II-0 (A), ADIII/IV-A (B), and middle-aged (MA) cases using gelatin sepharore 4B, and then separated in $10 \%$ SDS-PAGE gel containing $0.1 \%$ gelatin. The figure represents the negative image of the zymography gel showing a band of $72 \mathrm{kDa}$ corresponding to MMP2. A significant increase in MMP2 expression levels is seen in ADI/II- 0 ( $n=5 ; p=0.007)$ and ADIII/IV-A $(n=5, p=0.008)$ with respect to MA cases $(n=5)$. Open circle and triangles show values of individual cases. Filled shapes indicate mean \pm SD.

in order to study the distribution of the metalloproteinase with respect to the tau lesions (Fig. 3). MMP2 co-localizes with Tau-Thr181 in some NFTs and dystrophic neurites in cases with AD-related pathology.

\section{MMP2 cleaves recombinant tau at $C$-terminal region}

Full-length tau 4R (Tau40) was incubated with increasing concentrations of recombinant active MMP2 for $3 \mathrm{~h}$ at $37^{\circ} \mathrm{C}$. The pattern of tau cleavage was analyzed in $12 \%$ SDS-PAGE and the proteolysis bands were visualized with silver staining (Fig. 4A). A gradual increase in MMP2 concentrations from $50 \mathrm{ng}$ to $500 \mathrm{ng}$ reduced the intensity of the $55 \mathrm{kDa}$ band, corresponding to full-length tau (lane 1), while low molecular weight bands, resulting from tau cleavage, appeared in a dose-dependent manner (lanes 2-4). At the lowest concentration assayed (50 ng MMP2), three major bands of 35,30 , and $25 \mathrm{kDa}$ were observed, but the band at $30 \mathrm{kDa}$ become much more intense and new low molecular weight bands ranging from 25 to $10 \mathrm{kDa}$ appeared at higher concentrations of MMP2.

In order to validate tau cleavage by MMP2, the proteolytic bands were visualized with immunoblotting using $\mathrm{N}$-terminal or $\mathrm{C}$-terminal tau antibodies (Fig. 4B). The N-terminal antibody raised against the first 16 amino acids of tau showed a band of $55 \mathrm{kDa}$ corresponding to full-length tau and several proteolytic bands between 40 and $30 \mathrm{kDa}$ corresponding to truncated tau (Fig. 4B left, lane 1). Incubation with $200 \mathrm{ng}$ and $500 \mathrm{ng}$ of MMP2 reduced the expression of the upper bands and resulted in the appearance of intermediate bands of about $35 \mathrm{kDa}$ and below $20 \mathrm{kDa}$ (Fig. 4B left, lanes 3, 4). Additionally, immunoblotting with the C-terminal tau antibody showed some bands, ranging between 37 and $25 \mathrm{kDa}$, which were present in the baseline samples and after incubation with $50 \mathrm{ng}$ MMP2. Incubation with higher doses of MMP2 abolished C-terminal immunoreactivity thus indicating that major cleavage of tau occurs at the C-terminal domain. (Fig. 4B, right, comparing lanes 1, 2 with lanes 3 and 4).

\section{Lack of proteolysis of PHF tau by MMP2}

To further investigate whether MMP2 had an effect on tau aggregates in vivo, we analyzed MMP2 degradation of PHF-tau extracted from AD brains. Samples were analyzed with western blot using phospho-tau AT8 antibody and N-terminal and C-terminal (Tau46) antibodies (Fig. 5A, B, C, respectively). PHF-tau was treated either with or without $E$. coli alkaline phosphatase to obtain dephosphorylated and phosphorylated PHF-tau samples. Typical bands of 68 $\mathrm{kDa}, 64 \mathrm{kDa}$, and $60 \mathrm{kDa}$ [19] together with a higher band of $73 \mathrm{kDa}$ and several bands of low molecular weight, corresponding to truncated tau 


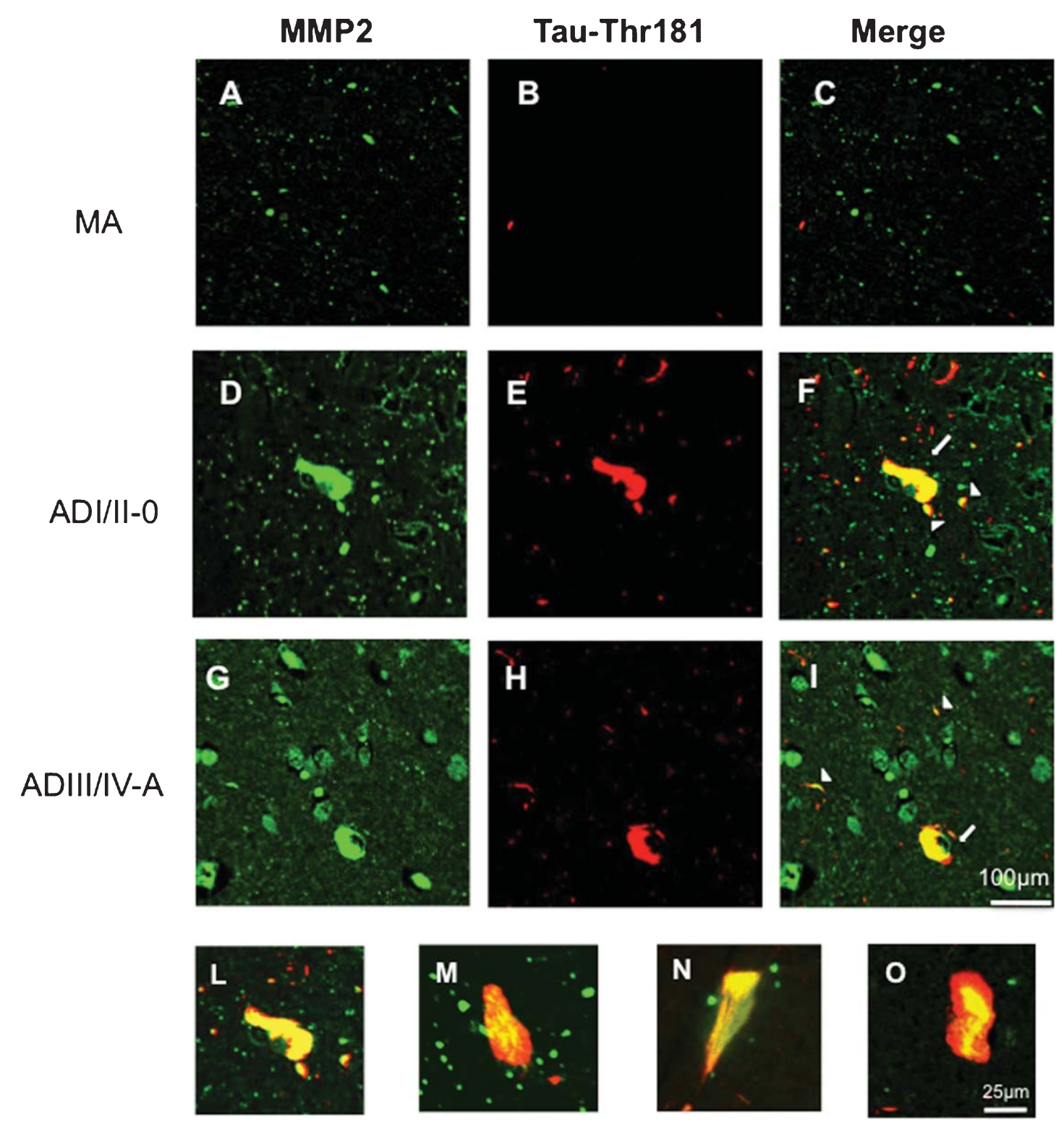

Fig. 3. Immunofluorescence and confocal microscopy of entorhinal cortex (layer II) of middle aged (MA) (A-C), ADI/II-0 (D-F), and ADIII/IV-A (G-I) cases show MMP2 immunoreactivity in neurons with NFTs (arrows) and some dystrophic neurites (arrowhead) in ADI/II-0 and ADIII/IV-A. A, D, G (green): MMP2 antibody; B, E, H (red): anti Tau-Thr181 antibody; C, F, I: merge. L-O: Detail of MMP2-Tau co-localization in tangles of cases of ADI/II-0 (L, M) and ADIII/IV-A (N, O).

species, were found in non-treated samples (not subjected to dephosphorylation Fig. 5A, in all panels). No signal of phospho-tau protein was seen in the dephosphorylated sample using the AT8 antibody (Fig. 5A; lane B).

Several bands ranging between 70 and $50 \mathrm{kDa}$ were observed instead following tau dephosphorylation (Fig. 5B, C; lanes B). Samples of PHF-tau treated either with or without $E$. coli phosphatase were then incubated with MMP2. Immunoblots with $\mathrm{N}$-terminal and C-terminal (Tau46) antibodies did not show any difference in the degradation pattern between MMP2-non-incubated and MMP2-incubated samples independently of the phosphorylation state of tau (Fig. 5B, C; lanes C, D). No additional PHF cleavage by MMP2 was observed in phosphorylated or dephosphorylated samples even at high exposition time of the membrane (Fig. 5B, C). 

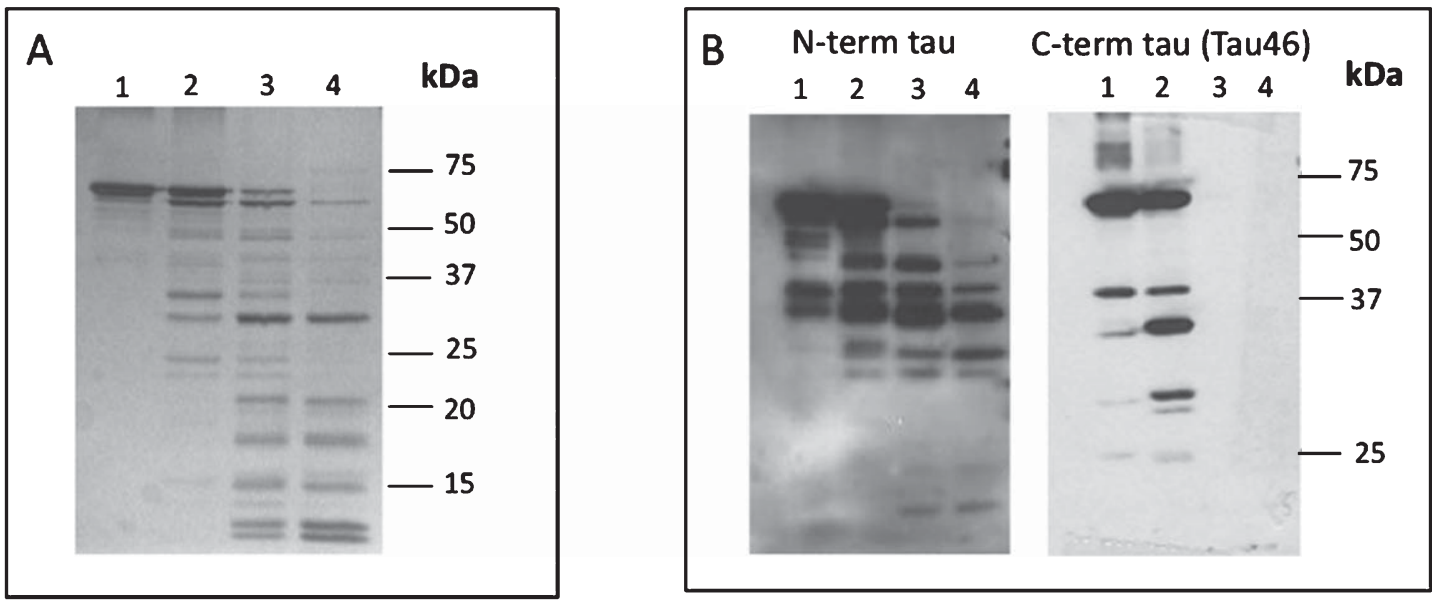

Fig. 4. A) MMP2 cleaves recombinant tau in a dose-dependent manner. MMP2 concentration from $50 \mathrm{ng}$ to $200 \mathrm{ng}$ and $500 \mathrm{ng}$ (lanes 2,3 , and 4 , respectively) reduces the intensity of the $55 \mathrm{kDa}$ band corresponding to full-length tau. Low molecular weight bands appear following tau incubation with increasing concentrations of MMP2; silver staining. B) Immunoblotting with antibodies against N-terminal tau and C-terminal tau (Tau46) shows that incubation of recombinant tau with MMP2 results in reduced levels of full-length tau and in the appearance of bands of lower molecular weight revealed with N-terminal tau antibody. In contrast, no lower bands appear with C-terminal tau antibody following incubation of recombinant tau with $200 \mathrm{ng}$ and $500 \mathrm{ng}$ of MMP2. Lane 1: tau alone; lanes 2, 3, 4: tau incubated with $50 \mathrm{ng}, 200 \mathrm{ng}$, and $500 \mathrm{ng}$ of MMP2, respectively.

AT8

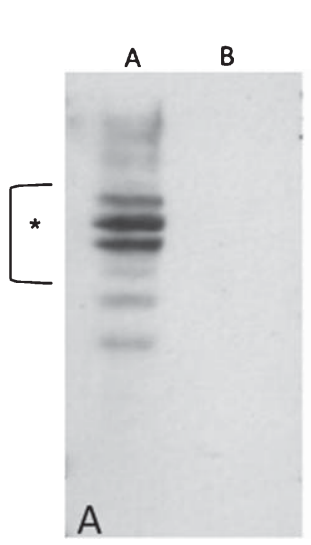

N-term tau

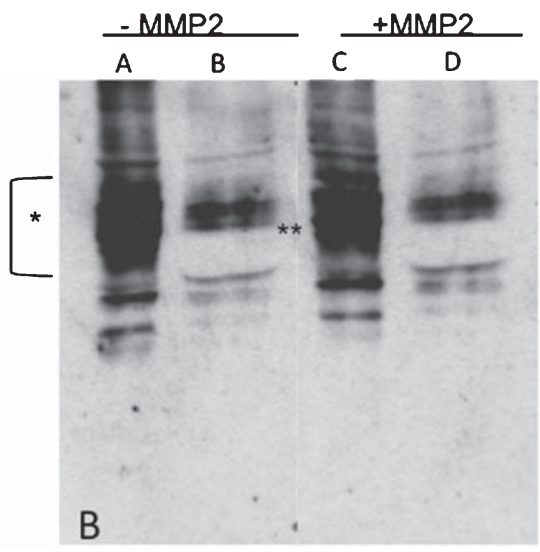

C-term tau (Tau46)

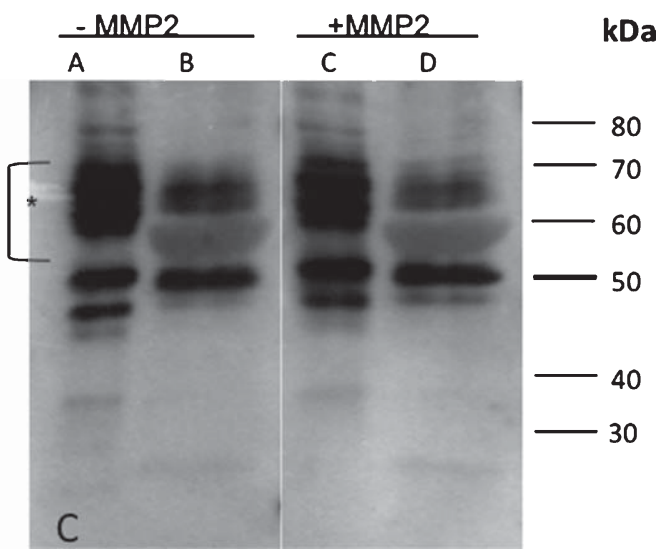

Fig. 5. PHF-tau was extracted from AD cases and half of the sample was treated with E. coli alkaline phosphatase (lanes B and D) while the other half was not subjected to phosphatase treatment (lanes A and C). Samples were analyzed with western blot using phospho-tau AT8 antibody, $\mathrm{N}$-terminal and C-terminal (Tau46) antibodies (A, B, and C, respectively). Samples without alkaline phosphatase pre-treatment showed the typical $\mathrm{AD}$ tau pattern of $68 \mathrm{kDa}, 64 \mathrm{kDa}$, and $60 \mathrm{kDa}$ bands together with several bands of lower molecular weight corresponding to truncated tau. This pattern is best visualized with short incubation time as seen with AT8 antibody (A, lane A). Longer incubation time, as employed with anti-N-terminal and C-terminal antibodies, results in a blurred band due to overexposure (B and C, lanes A, asterisk). Yet overexposure is needed to better visualize the effects of MMP2 incubation (lanes C and D). Hyperphosphorylated tau band pattern is modified following pre-treatment with alkaline phosphatase: no bands are seen using AT8 antibody (A, lane B), whereas several bands ranging between 70 and 50 $\mathrm{kDa}$ are identified with $\mathrm{N}$-terminal and $\mathrm{C}$-terminal antibodies. (B and $\mathrm{C}$, lanes $\mathrm{B}$; double asterisk). Immunoblots with $\mathrm{N}$-terminal and $\mathrm{C}$-terminal (Tau46) antibody show no modifications of the profile of tau bands, following incubation with MMP2 (B and C; lanes C, D).

\section{DISCUSSION}

The present study, using western blotting and gelatin zymography, shows increased expression levels of MMP2 at early (Braak and Braak I/II-0) and middle
(Braak and Braak III/IV-A) stages of AD-related pathology compared with middle-aged cases with no AD-related pathology. Since the mean age in MA cases was lower than in cases with AD-related pathology, it can be suggested that increased MMP2 expression 
levels might be the effect of aging rather than the effect of AD pathology. However, MMP2 was found in some neurons with NFTs and in dystrophic neurites, co-localizing with hyperphosphorylated tau, as visualized with double-labeling immunofluorescence and confocal microscopy, thus indicating a close relationship between MMP2 and hyper-phosphorylated tau in AD.

Proteolysis of tau contributes to tangle formation [20-22]. An important fraction of tau in fibrils is truncated at positions Glu-391 and Asp-421, and tau is cleaved at Asp-421 by caspases [23]. Removal of the C-terminal domain of tau enhances nucleation and fibrillization reactions in vitro [24]. Moreover, time-course analysis and antibody labeling of tau inclusions demonstrates that tau oligomers appear early in the AD pathogenesis and are the toxic form of tau, as they are able to inhibit axonal transport in neurons and are involved in synapse and neuron loss [25-27].

So far, MMPs have always been considered to be only extracellular degrading enzymes, and their substrates have always been identified in proteins of the extracellular matrix. However, MMP2 has intracellular proteolytic activity in variegated pathological conditions such as tumor growth and heart and brain ischemia [28]. MMP9 is able to cleave tau at N- and C-terminal regions, thus liberating the hexapeptide domain of the protein and facilitating tau aggregation [17]. The present study demonstrates that MMP2 is able to cleave tau in vitro after co-incubation of recombinant full-length tau with MMP2; western blotting further shows that tau fragments are likely generated at the $\mathrm{C}$-terminal region of the protein. Based on these evidences, overexpression of MMP2 in ADI/II and ADIII/IV at neurofibrillary lesions, may suggest a role of MMP2 in the promotion of tau fibrillization.

It may also be suggested that MMP2 has physiological functions in normal tau proteolysis whatever tau degradation occurs in the extracellular space or in the cytoplasm of neurons.

Thrombin and pro-thrombin are found in NFTs, dystrophic neurites, and reactive microglia [29], thus suggesting that they may be involved in tau proteolysis. However, increased tau phosphorylation inhibits this process, and PHFs are more resistant to thrombin cleavage [30] in the same line as we can here observed for MMP2. We can then conclude that upregulation of MMP2 at early and middle stages of AD-related pathology may be an additional mechanism which promote tau fibrillization. Moreover, the incapacity of MMP2 to degrade hyperphosphorylated tau in paired helical filaments may further contribute to the accumulation of tau aggregates.

\section{ACKNOWLEDGMENTS}

This study was funded by the Seventh Framework Programme of the European Commission, grant agreement 278486: DEVELAGE and FIS grant: PI 14-757. Beatrice Terni was recipient of a Juan de la Cierva fellowship from the Ministry of Economy and Competiveness (MINECO). We thank T. Yohannan for editorial help. There is no conflict of interest including any financial, personal or other relationships with other people or organizations within the three years from the beginning of the work. Brain samples were obtained from the Institute of Neuropathology Brain Bank following the guidelines and approval of the local ethics committee.

Authors' disclosures available online (http://j-alz. com/manuscript-disclosures/14-2460r1).

\section{SUPPLEMENTARY MATERIAL}

The supplementary material is available in the electronic version of this article: http://dx.doi.org/ 10.3233/JAD-142460.

\section{REFERENCES}

[1] Duyckaerts C, Dickson D (2011) Neuropathology of Alzheimer's disease. In Neurodegeneration: The Molecular Pathology of Dementia and Movement Disorders, Dickson D, Weller R, eds. Wiley-Blackwell, Oxford, pp. 62-91.

[2] Ferrer I (2012) Defining Alzheimer as a common age-related neurodegenerative process not inevitably leading to dementia. Prog Neurobiol 97, 38-51.

[3] Braak H, Braak E (1991) Neuropathological stageing of Alzheimer-related changes. Acta Neuropathol 82, 239-259.

[4] Zempel H, Mandelkow E (2014) Lost after translation: missorting of tau protein and consequences for Alzheimer's disease. Trends Neurosci 37, 721-732.

[5] Selkoe DJ (2008) Biochemistry and molecular biology of amyloid beta-protein and the mechanism of Alzheimer's disease. Handb Clin Neurol 89, 245-260.

[6] Stetler-Stevenson WG (1996) Dynamics of matrix turnover during pathologic remodeling of the extracellular matrix. Am $J$ Pathol 148, 1345-1350.

[7] Lo E, Wang X, Cuzner M (2002) Extracellular proteolysis in brain injury and inflammation: role for plasminogen activators and matrix metalloproteinases. J Neurosci Res 69, 1-9.

[8] Backstrom J, Lim G, Cullen M, Tökés Z (1996) Matrix metalloproteinase-9 (MMP-9) is synthesized in neurons of the human hippocampus and is capable of degrading the amyloid- $\beta$ peptide (1-40). J Neurosci 16, 7910-7919.

[9] LePage R, Fosang A, Fuller S, Murphy G, Evin G, Beyreuther K, Masters C, Small D (1995) Gelatinase A possesses a $\beta$-secretase-like activity in cleaving the amyloid 
protein precursor of Alzheimer's disease. FEBS Lett 377, 267-270.

[10] Miyazaki K, Hasegawa M, Funahashi K, Umeda M (1993) A metalloproteinase inhibitor domain in Alzheimer amyloid protein precursor. Nature 362, 839-841.

[11] White AR, Du T, Laughton KM, Volitakis I, Sharples RA, Xilinas ME, Hoke DE, Holsinger RM, Evin G, Cherny RA, Hill AF, Barnham KJ, Li QX, Bush AI, Masters CL (2006) Degradation of the Alzheimer disease amyloid $\beta$-peptide by metal-dependent up-regulation of metalloprotease activity. J Biol Chem 281, 17670-17680.

[12] Deb S, Gottschall PE (1996) Increased production of matrix metalloproteinases in enriched astrocyte and mixed hippocampal cultures treated with $\beta$-amyloid peptides. J Neurochem 66, 1641-1647.

[13] Jung S, Zhang W, Van Nostrand W (2003) Pathogenic $\mathrm{A} \beta$ induces the expression and activation of matrix metalloproteinase-2 in human cerebrovascular smooth muscle cells. J Neurochem 85, 1208-1215.

[14] Yin KJ, Cirrito JR, Yan P, Hu X, Xiao Q, Pan X, Bateman R, Song H, Hsu FF, Turk J, Xu J, Hsu CY, Mills JC, Holtzman DM, Lee JM (2006) Matrix metalloproteinases expressed by astrocytes mediate extracellular amyloid- $\beta$ peptide catabolism. J Neurosci 26, 10939-10948.

[15] Bruno MA, Mufson EJ, Wuu J, Cuello AC (2009) Increased matrix metalloproteinase 9 activity in mild cognitive impairment. J Neuropathol Exp Neurol 68, 1309-1318.

[16] Py NA, Bonnet AE, Bernard A, Marchalant Y, Charrat E, Checler F, Khrestchatisky M, Baranger K, Rivera S (2014) Differential spatio-temporal regulation of MMPs in the 5xFAD mouse model of Alzheimer's disease: evidence for a pro-amyloidogenic role of MT1-MMP. Front Aging Neurosci 6, 247.

[17] Nübling G, Levin J, Bader B, Israel L, Bötzel K, Lorenzl S, Giese A (2012) Limited cleavage of tau with matrixmetalloproteinase MMP-9, but not MMP-3, enhances tau oligomer formation. Exp Neurol 237, 470-476.

[18] Braak H, Alafuzoff I, Arzberger T, Kretzschmar H, Del Tredici K (2006) Staging of Alzheimer disease-associated neurofibrillary pathology using paraffin sections and immunocytochemistry. Acta Neuropathol 112, 389-404.

[19] Goedert M, Spillantini MG, Cairns NJ, Crowther RA (1992) Tau proteins of Alzheimer paired helical filaments: abnormal phosphorylation of all six brain isoforms. Neuron $\mathbf{8}$, 159-168.

[20] Gamblin TC, Chen F, Zambrano A, Abraha A, Lagalwar S, Guillozet AL, Lu M, Fu Y, Garcia-Sierra F, LaPointe N, Miller R, Berry RW, Binder LI, Cryns VL (2003) Caspase cleavage of tau: linking amyloid and neurofibrillary tangles in Alzheimer's disease. Proc Natl Acad Sci U S A 100, 1003210037.

[21] Guillozet-Bongaarts AL, Garcia-Sierra F, Reynolds MR, Horowitz PM, Fu Y, Wang T, Cahill ME, Bigio EH, Berry RW, Binder LI (2005) Tau truncation during neurofibrillary tangle evolution in Alzheimer's disease. Neurobiol Aging 26, 1015-1022.

[22] Hanger DP, Wray S (2010) Tau cleavage and tau aggregation in neurodegenerative disease. Biochem Soc Trans 38, 10161020.

[23] Rissman RA, Poon WW, Blurton-Jones M, Oddo S, Torp R, Vitek MP, LaFerla FM, Rohn TT, Cotman CW (2004) Caspase-cleavage of tau is an early event in Alzheimer disease tangle pathology. J Clin Invest 114, 121-130.

[24] Berry RW, Abraha A, Lagalwar S, LaPointe N, Gamblin TC, Cryns VL, Binder LI (2003) Inhibition of tau polymerization by its carboxy-terminal caspase cleavage fragment. Biochemistry 42, 8325-8331.

[25] Wang Y, Garg S, Mandelkow EM, Mandelkow E (2010) Proteolytic processing of tau. Biochem Soc Trans 38, 955-961.

[26] Takashima A (2010) Tau aggregation is a therapeutic target for Alzheimer's disease. Curr Alzheimer Res 7, 665-669.

[27] Ward SM, Himmelstein DS, Lancia JK, Binder LI (2012) Tau oligomers and tau toxicity in neurodegenerative disease. Biochem Soc Trans 40, 667-671.

[28] Solli AI, Fadnes B, Winberg JO, Uhlin-Hansen L, HadlerOlsen E (2013) Tissue- and cell-specific co-localization of intracellular gelatinolytic activity and matrix metalloproteinase 2. J Histochem Cytochem 61, 444-461.

[29] Arai T, Miklossy J, Klegeris A, Guo JP, McGeer PL (2006) Thrombin and prothrombin are expressed by neurons and glial cells and accumulate in neurofibrillary tangles in Alzheimer disease brain. J Neuropathol Exp Neurol 65, 19-25.

[30] Arai T, Guo JP, McGeer PL (2005) Proteolysis of nonphosphorylated and phosphorylated tau by thrombin. J Biol Chem 280, 5145-5153. 